\title{
Inhomogeneous M-Theory Cosmologies
}

\author{
Alan A. Coley \\ Department of Mathematics and Statistics, \\ Dalhousie University, Halifax, N.S.,B3H 3J5, Canada \\ R. J. van den Hoogen! \\ Department of Mathematics, Statistics, \\ and Computer Science, Saint Francis Xavier University, \\ Antigonish, N.S., B2G 2W5, Canada
}

\begin{abstract}
We study a class of inhomogeneous and anisotropic $G_{2}$ string cosmological models. In the case of separable $G_{2}$ models we show that the governing equations reduce to a system of ordinary differential equations. We focus on a class of separable $G_{2}$ M-theory cosmological models, and study their qualitative behaviour (a class of models with time-reversed dynamics is also possible). We find that generically these inhomogeneous M-theory cosmologies evolve from a spatially inhomogeneous and negatively curved model with a non-trivial form field towards spatially flat and spatially homogeneous dilaton-moduli-vacuum solutions with trivial form-fields. The late time behaviour is the same as that of spatially homogeneous models previously studied. However, the inhomogeneities are not dynamically insignificant at early times in these models.
\end{abstract}

*Electronic address: aac@mscs.dal.ca

$\dagger$ Electronic address: rvandenh@stfx.ca 


\section{STRING COSMOLOGY}

Non-perturbative M-theory encompasses and unifies all five anomaly-free, perturbative superstring theories [1] and corresponds to eleven-dimensional supergravity in the lowenergy limit [2]. In particular, the compactification of $\mathrm{M}$-theory on a circle, $S^{1}$, leads to the type IIA superstring. A study of the qualitative cosmological effects that can arise in M-theory is therefore of considerable importance. To lowest order (in the inverse string tension), the tree-level effective action for massless fields contains a dilaton, a form field (which in 4-dimensions is dynamically dual to pseudoscalar axion field) and (a) stringy cosmological constant(s). Even in this approximation the one-loop string equations of motion for inhomogeneous backgrounds are very difficult to solve, and it is a useful first step to consider models in which the homogeneity is broken only in one spatial direction. Metrics that admit two commuting (orthogonally-transitive) space-like Killing vectors are referred to as $G_{2}$ spacetimes.

String models admitting an abelian group, $G_{2}$, of isometries have a number of important physical applications. The spatially homogeneous Bianchi types $\mathrm{I}_{-} \mathrm{VII}_{h}$ and locally rotationally symmetric (LRS) types VIII and IX admit a $G_{2}$ group of isometries [3] and so the $G_{2}$ cosmologies can be considered as inhomogeneous generalizations of these Bianchi models. Non-linear inhomogeneities in the dilaton and axion fields can be investigated and, in principle, this allows density perturbations in string-inspired inflationary models such as the pre-big bang scenario to be studied [4, 5]. Given the potential importance of this scenario it is important to study its generality with respect to inhomogeneities as well with respect to anisotropies. The general effects of small inhomogeneities and anisotropies have been studied by Veneziano [4.

In general relativity (GR) the generic singularity is neither spatially homogeneous or isotropic. Hence it is of interest to study more general models. In particular, it has been conjectured that $G_{2}$ metrics represent a first approximation to the general solution of Einstein gravity in the vicinity of a curvature singularity [6, 7, 8, 9]. The high curvature regime

is precisely the regime where stringy deviations from GR are expected to be significant. The $G_{2}$ models studied here may therefore provide insight into the generic behaviour of cosmologies at very early times.

A number of exact inhomogeneous and anisotropic $G_{2}$ string cosmologies have been found. 
Barrow and Kunze studied an inhomogeneous generalization of the Bianchi type I string cosmology [10 and Feinstein, Lazkoz and Vazquez-Mozo derived a closed, inhomogeneous model by applying duality transformations on the LRS Bianchi type IX cosmology [11. Clancy et al. have found inhomogeneous generalizations of the Bianchi type $\mathrm{VI}_{h}$ universe and have studied their asymptotic behaviour [12].

In general, the field equations reduce to a system of coupled, partial differential equations in two variables when spatial homogeneity is broken along a single direction. Unfortunately, these equations are still very complicated. However, solutions can be found due to the non-compact global symmetries of the string effective action. When the metric admits two commuting space-like Killing vectors, there exists an infinite-dimensional symmetry on the space of solutions that may be identified infinitesimally with the $\mathrm{O}(2,2)$ current algebra [13, 14, 15]. This symmetry reduces to the Geroch group, corresponding to the $\operatorname{SL}(2, R)$ current algebra, when the dilaton and two-form potential are trivial [16], and includes the global $\mathrm{SL}(2, R) \mathrm{S}$-duality of the action.

New inhomogeneous $G_{2}$ string cosmologies containing a non-trivial two-form potential may be generated by an application of both the $\mathrm{S}$ - and $\mathrm{T}$-duality symmetries from simpler (dilaton-vacuum) seed solutions. Lidsey et al. [17] discuss the non-compact, global symmetries of the string effective action in a variety of settings and review various methods for solving the Einstein-scalar field equations utilizing generating techniques (from solutions with a minimally coupled, massless scalar field from a vacuum, $G_{2}$ cosmology). In particular, Feinstein, Lazkoz and Vázquez-Mozo [11] present an algorithm which permits the construction of inhomogeneous string solutions by employing a Buscher transformation, inverse scattering techniques [0, [8], followed by the generating technique of Wainwright, Ince and Marshman [18]. Feinstein et al. employ this algorithm to generate a closed, inhomogeneous string cosmology with $S^{3}$ topology from a LRS Bianchi type IX solution [11, 19]. However, this algorithm involves a number of non-trivial operations, and an alternative and more straightforward approach is to apply an $\mathrm{O}(2,2)$ transformation directly to the seed cosmology [17].

In this paper we shall consider a class of separable string cosmological models whose governing equations reduce to ordinary differential equations (ODE) which can be studied by qualitative methods. In particular, we shall focus on a class of M-theory cosmological models. 


\section{STRING ACTION}

We consider the general string action in the form 20]

$$
S=\int d^{4} x \sqrt{-g}\left\{e^{-\Phi}\left[R+(\nabla \Phi)^{2}-6(\nabla \beta)^{2}-\frac{1}{2} e^{2 \Phi}(\nabla \sigma)^{2}-2 \Lambda\right]-\frac{1}{2} Q^{2} e^{-6 \beta}-\Lambda_{\mathrm{M}}\right\},
$$

in terms of the pseudo-scalar axion field, $\sigma$, the 4-D dilaton field $\Phi$, and the modulus field $\beta$, where $\Lambda$ and $\Lambda_{M}$ represent cosmological constant terms and $Q^{2}$ may be interpreted as a 0 -form field strength. This is a phenomenological action representing the bosonic sector of the effective supergravity action for the low-energy limit of M-theory and encompasses other string theories [20]. We are particularly interested in the class of four-dimensional cosmologies derived from the type IIA string and M-theory effective actions and which include a non-trivial Ramond-Ramond (RR) sector [21]. In these models a specific compactification from eleven to four dimensions was considered, where the topology of the internal dimensions was assumed to be a product space consisting of a circle and an isotropic six-torus [21]; this is dynamically equivalent to compactifications on a Calabi-Yau three-fold [23]. The FRW models in this class of cosmologies was studied in 21]

Defining

$$
\mathcal{L}_{\mathrm{M}}=-3 e^{-\Phi}(\nabla \beta)^{2}-\frac{1}{4} e^{\Phi}(\nabla \sigma)^{2}-\Lambda e^{-\Phi}-\frac{1}{4} Q^{2} e^{-6 \beta}-\frac{1}{2} \Lambda_{\mathrm{M}} ; \quad T_{\alpha \beta} \equiv g_{\alpha \beta} \mathcal{L}_{\mathrm{M}}-2 \frac{\partial \mathcal{L}_{\mathrm{M}}}{\partial g^{\alpha \beta}}
$$

the Euler-Lagrange equations then lead to the field equations (FE) 24]

$$
\begin{aligned}
G_{\mu \nu}= & -\nabla_{\mu} \nabla_{\nu} \Phi+6 \nabla_{\mu} \beta \nabla_{\nu} \beta+\frac{1}{2} e^{2 \Phi} \nabla_{\mu} \sigma \nabla_{\nu} \sigma \\
& -\frac{1}{2} g_{\mu \nu}\left[(\nabla \Phi)^{2}+6(\nabla \beta)^{2}+\frac{1}{2} e^{2 \Phi}(\nabla \sigma)^{2}+2 \Lambda+\frac{1}{2} Q^{2} e^{\Phi-6 \beta}\right. \\
& \left.+\Lambda_{\mathrm{M}} e^{\Phi}-2 \square \Phi\right], \\
\square \Phi= & \frac{1}{2}(\nabla \Phi)^{2}+3(\nabla \beta)^{2}-\frac{1}{4} e^{2 \Phi}(\nabla \sigma)^{2}+\Lambda-\frac{1}{2} R, \\
\square \beta= & \nabla_{\mu} \Phi \nabla^{\mu} \beta-\frac{1}{4} Q^{2} e^{\Phi-6 \beta}, \\
\square \sigma= & -\nabla_{\mu} \Phi \nabla^{\mu} \sigma .
\end{aligned}
$$

In the above, greek indices take on values $0,1,2,3$, and units are chosen so that $16 \pi \hat{G}=1$. 


\section{III. $G_{2}$ COSMOLOGIES}

Let us examine (2.2) within the context of $G_{2}$ cosmological models described by the line element

$$
d s^{2}=e^{2 F}\left(-d t^{2}+d z^{2}\right)+e^{G}\left(e^{q} d x^{2}+e^{-q} d y^{2}\right)
$$

where the metric functions $\{F, G, q\}$ and the string functions $\{\Phi, \beta, \sigma\}$ are all functions

of $t$ and $z$ only. For any $q(t, z)$, we define $\dot{q} \equiv \frac{\partial q}{\partial t}, q^{\prime} \equiv \frac{\partial q}{\partial z}$, and $\Delta^{2} q \equiv \ddot{q}-q^{\prime \prime}$. The local behaviour of these models is determined by the gradient $B_{\mu} \equiv \partial_{\mu} G$, and cosmological solutions arise if $B_{\mu}$ is globally time-like.

Also, the Ricci scalar is given by

$$
R=\frac{1}{2} e^{-2 F}\left[4 \Delta^{2} G+4 \Delta^{2} F+3\left(\dot{G}^{2}-G^{\prime 2}\right)+\left(\dot{q}^{2}-q^{\prime 2}\right)\right] .
$$

Using these expressions, and defining the modified dilaton field,

$$
\varphi \equiv \Phi-F-G
$$


the field equations become

$$
\begin{aligned}
& \Delta^{2} \varphi=\frac{1}{2}\left[(\dot{\varphi}+\dot{F})^{2}-\left(\varphi^{\prime}+F^{\prime}\right)^{2}\right]+\frac{1}{4}\left(\dot{G}^{2}-G^{\prime 2}\right)+\frac{1}{4}\left(\dot{q}^{2}-q^{\prime 2}\right) \\
& +3\left(\dot{\beta}^{2}-\beta^{\prime 2}\right)-\frac{1}{4} e^{2 \varphi+2 F+2 G}\left(\dot{\sigma}^{2}-\sigma^{\prime 2}\right)-\Lambda e^{2 F}, \\
& \Delta^{2} \beta=\left[(\dot{\varphi}+\dot{F}) \dot{\beta}-\left(\varphi^{\prime}+F^{\prime}\right) \beta^{\prime}\right]+\frac{1}{4} Q^{2} e^{\varphi+3 F+G-6 \beta}, \\
& \Delta^{2} \sigma=-\left[(\dot{\varphi}+\dot{F}+2 \dot{G}) \dot{\sigma}-\left(\varphi^{\prime}+F^{\prime}+2 G^{\prime}\right) \sigma^{\prime}\right] \\
& \Delta^{2} q=\left[(\dot{\varphi}+\dot{F}) \dot{q}-\left(\varphi^{\prime}+F^{\prime}\right) q^{\prime}\right] \\
& \Delta^{2} F=\frac{1}{2}(\dot{\varphi}+\dot{F})^{2}-\frac{1}{2}\left(\varphi^{\prime}+F^{\prime}\right)^{2}-\frac{1}{4}\left(\dot{G}^{2}-G^{\prime 2}\right)-\frac{1}{4}\left(\dot{q}^{2}-q^{\prime 2}\right) \\
& -3\left(\dot{\beta}^{2}-\beta^{2}\right)+\frac{1}{4} e^{2 \varphi+2 F+2 G}\left(\dot{\sigma}^{2}-\sigma^{\prime 2}\right)-\Lambda e^{2 F} \\
& -\frac{1}{2} Q^{2} e^{\varphi+3 F+G-6 \beta}-\Lambda_{\mathrm{M}} e^{\varphi+3 F+G}, \\
& \Delta^{2} G=\left[(\dot{\varphi}+\dot{F}) \dot{G}-\left(\varphi^{\prime}+F^{\prime}\right) G^{\prime}\right]+\left(\dot{\sigma}^{2}-\sigma^{\prime 2}\right) e^{2 \varphi+2 F+2 G} \\
& -\frac{1}{2} Q^{2} e^{\varphi+3 F+G-6 \beta}-\Lambda_{\mathrm{M}} e^{\varphi+3 F+G}, \\
& (\dot{\varphi}+\dot{F})^{\cdot}+\left(\varphi^{\prime}+F^{\prime}\right)^{\prime}=2(\dot{\varphi}+\dot{F}) \dot{F}+2\left(\varphi^{\prime}+F^{\prime}\right) F^{\prime}+\frac{1}{2}\left(\dot{G}^{2}+G^{\prime 2}\right) \\
& +\frac{1}{2}\left(\dot{q}^{2}+q^{\prime 2}\right)+6\left(\dot{\beta}^{2}+\beta^{\prime 2}\right)+\frac{1}{2}\left(\dot{\sigma}^{2}+\sigma^{\prime 2}\right) e^{2 \varphi+2 F+2 G}, \\
& (\dot{\varphi}+\dot{F})^{\prime}=\frac{1}{2} \dot{G} G^{\prime}+\frac{1}{2} \dot{q} q^{\prime}+6 \dot{\beta} \beta^{\prime}+F^{\prime}(\dot{\varphi}+\dot{F})+\dot{F}\left(\varphi^{\prime}+F^{\prime}\right) \\
& +\frac{1}{2} e^{2 \varphi+2 F+2 G} \dot{\sigma} \sigma^{\prime}
\end{aligned}
$$

These equations reduce to those in 20, 21] in the appropriate limits. 


\section{SEPARABLE $G_{2}$ STRING COSMOLOGIES}

\section{A. General Case}

Let us assume separability of the metric functions of the form

$$
\begin{aligned}
F(t, z) & \equiv F(t)+f(z), \\
G(t, z) & \equiv G(t)+g(z), \\
q(t, z) & \equiv q(t)+\nu(z),
\end{aligned}
$$

and appropriate separability conditions on the matter fields $\Phi(t, z), \beta(t, z), \sigma(t, z)$. Then the Ricci Scalar is given by

$$
R=\frac{1}{2} e^{-2 F-2 f}\left[4 \ddot{G}+4 \ddot{F}+3 \dot{G}^{2}+\dot{q}^{2}-\left(4 g^{\prime \prime}+4 f^{\prime \prime}+3 g^{\prime 2}+\nu^{\prime 2}\right)\right] .
$$

If

$$
4 g^{\prime \prime}+4 f^{\prime \prime}+3 g^{\prime 2}+\nu^{\prime 2}=C
$$

where $C$ is a constant, then we obtain a condition which constrains the spatial dependence of the metric. The Ricci Scalar is then given by

$$
R=\frac{1}{2} e^{-2 F-2 f}\left[4 \ddot{G}+4 \ddot{F}+3 \dot{G}^{2}+\dot{q}^{2}-C\right] .
$$

Putting this expression for the Ricci Scalar into the action (2.1), the spatial dependence of the geometrical terms can be eliminated (by integration over the spatial coordinates in the action). After applying any further separability conditions (on the matter fields), the resulting FE will be a system of ODEs. Note that the effect of the spatial dependence is to add a further contribution $(C)$ to the cosmological constant $\Lambda$ in the action. 


\section{B. Specific Example: Linear Dependence in $z$}

In an attempt to remove the $z$-dependence, let us assume separability of the form

$$
\begin{aligned}
F(t, z) & \equiv F(t)+\frac{1}{2} c z, \\
G(t, z) & \equiv G(t) \\
q(t, z) & \equiv q(t)+a z \\
\Phi(t, z) & \equiv \Phi(t)+m z \\
\beta(t, z) & \equiv \beta(t)+n z \\
\sigma(t, z) & \equiv \sigma(t)+l z
\end{aligned}
$$

where $a, c, l, m, n$ are constants ( $a^{2}$ is equivalent to the constant $C$ in equation (4.1)), and therefore

$$
\varphi(t, z)=\Phi(t)-F(t)-G(t)+\left(m-\frac{1}{2} c\right) z \equiv \varphi(t)+\left(m-\frac{1}{2} c\right) z
$$

With the above assumptions, the metric becomes an extension of the inhomogeneous scalarfield $G_{2}$ solutions found by Feinstein and Ibanez [22] to M-Theoretical models. In addition, for particular values of the parameters, the metric reduces to spatially homogeneous Bianchi 
$I, I I I$ and $V I_{0}$ models. Hence,

$$
\begin{aligned}
\ddot{\varphi}= & \frac{1}{2}(\dot{\varphi}+\dot{F})^{2}+\frac{1}{4} \dot{G}^{2}+\frac{1}{4} \dot{q}^{2}+3 \dot{\beta}^{2}-\frac{1}{4} e^{2 \varphi+2 F+2 G+2 m z}\left(\dot{\sigma}^{2}-l^{2}\right) \\
& -\Lambda e^{2 F+c z}-\frac{1}{4}\left(a^{2}+2 m^{2}+12 n^{2}\right), \\
\ddot{\beta}= & (\dot{\varphi}+\dot{F}) \dot{\beta}+\frac{1}{4} Q^{2} e^{[\varphi+3 F+G-6 \beta+(c+m-6 n) z]}-m n, \\
\ddot{\sigma}= & -(\dot{\varphi}+\dot{F}+2 \dot{G}) \dot{\sigma}+m l, \\
\ddot{q}= & (\dot{\varphi}+\dot{F}) \dot{q}-m a, \\
\ddot{F}= & \frac{1}{2}(\dot{\varphi}+\dot{F})^{2}-\frac{1}{4} \dot{G}^{2}-\frac{1}{4} \dot{q}^{2}-3 \dot{\beta}^{2}+\frac{1}{4} e^{2 \varphi+2 F+2 G+2 m z}\left(\dot{\sigma}^{2}-l\right) \\
& -\Lambda e^{2 F+c z}-\frac{1}{2} Q^{2} e^{[\varphi+3 F+G-6 \beta+(c+m-6 n) z]}-\Lambda_{\mathrm{M}} e^{\varphi+3 F+G+(c+m) z} \\
& +\frac{1}{4}\left(a^{2}-2 m^{2}+12 n^{2}\right), \\
\ddot{G}= & (\dot{\varphi}+\dot{F}) \dot{G}+\left(\dot{\sigma}^{2}-l^{2}\right) e^{2 \varphi+2 F+2 G+2 m z} \\
& -\frac{1}{2} Q^{2} e^{[\varphi+3 F+G-6 \beta+(c+m-6 n) z]}-\Lambda_{\mathrm{M}} e^{\varphi+3 F+G+(c+m) z}, \\
(\ddot{F}+\ddot{\varphi})=2 & (\dot{F}+\dot{\varphi}+m)\left(\dot{F}+\frac{1}{2} c\right)+\frac{1}{2} \dot{G}^{2} \\
& +\frac{1}{2}(\dot{q}+a)^{2}+6(\dot{\beta}+n)^{2}+\frac{1}{2}(\dot{\sigma}+l)^{2} e^{2 \varphi+2 F+2 G+2 m z} .
\end{aligned}
$$

Note that the constraint equation can be rewritten as:

$$
\begin{aligned}
0= & (\dot{F}+m)^{2}-\dot{\varphi}^{2}+c(\dot{F}+\dot{\varphi}+m)+\frac{1}{2} \dot{G}^{2}+\frac{1}{2}(\dot{q}+a)^{2} \\
& +6(\dot{\beta}+n)^{2}+\frac{1}{2}(\dot{\sigma}+l)^{2} e^{2 \varphi+2 F+2 G+2 m z} \\
& +\Lambda e^{2 F+c z}+\frac{1}{2} Q^{2} e^{[\varphi+3 F+G-6 \beta+(c+m-6 n) z]}+\Lambda_{\mathrm{M}} e^{\varphi+3 F+G+(c+m) z} .
\end{aligned}
$$

In order for the $\mathrm{FE}$ to be independent of $z$, it is necessary that $m=0$ and that either $c=0$ or $c=6 n$. Furthermore if $c=0$ then we have that either $n=0$ or $Q=0$. In the $c=6 n$ case, we have that $\Lambda=\Lambda_{\mathrm{M}}=0$. It is the latter case that is of interest to us here. From here forth we shall assume that $c=6 n$ and that $\Lambda=\Lambda_{\mathrm{M}}=0$. This particular subcase, which is of relevence in M-theory cosmology, is of special physical interest. (The resulting FE in the remaining cases are displayed in [24]). 


\section{INHOMOGENEOUS M-THEORY COSMOLOGICAL MODELS}

Substituting $c=6 n, m=0, \Lambda=0, \Lambda_{M}=0$ into (3.4), (4.3) (and taking the linear combination $[(4.2 \mathrm{~g})-(4.2 \mathrm{~g})-(4.2 \mathrm{a})])$ we obtain the following system of ODE with two constraints:

$$
\begin{aligned}
& \ddot{\varphi}=\frac{1}{4}\left(\dot{q}^{2}+\dot{G}^{2}-a^{2}+2(\dot{\varphi}+\dot{F})^{2}+12 \dot{\beta}^{2}-12 n^{2}\right. \\
& \left.+e^{2 \varphi+2 F+2 G}\left(l^{2}-\dot{\sigma}^{2}\right)\right) \\
& \ddot{\beta}=\dot{\beta}(\dot{\varphi}+\dot{F})+\frac{1}{4} Q^{2} e^{-6 \beta+\varphi+3 F+G} \\
& \ddot{\sigma}=-\dot{\sigma}(\dot{\varphi}+\dot{F}+2 \dot{G}) \\
& \ddot{q}=\dot{q}(\dot{\varphi}+\dot{F}) \\
& \ddot{F}=\frac{1}{4}\left(3 a^{2}+\dot{q}^{2}+\dot{G}^{2}+36 n^{2}+12 \dot{\beta}^{2}+e^{2 \varphi+2 F+2 G}\left(l^{2}+3 \dot{\sigma}^{2}\right)\right. \\
& \left.-2(\dot{\varphi}-\dot{F})^{2}+8 \dot{F}^{2}\right) \\
& \ddot{G}=\dot{G}(\dot{\varphi}+\dot{F})-e^{2 \varphi+2 F+2 G}\left(l^{2}-\dot{\sigma}^{2}\right)-\frac{1}{2} Q^{2} e^{-6 \beta+\varphi+3 F+G} \\
& 0=2 \dot{\varphi}^{2}-\dot{G}^{2}-\dot{q}^{2}-12 \dot{\beta}^{2}-2 \dot{F}^{2}-12 n^{2}-a^{2} \\
& -e^{2 \varphi+2 F+2 G}\left(l^{2}+\dot{\sigma}^{2}\right)-Q^{2} e^{-6 \beta+\varphi+3 F+G} \\
& 0=a \dot{q}+l e^{2 \varphi+2 F+2 G} \dot{\sigma}+6 n(\dot{\varphi}+\dot{F}+2 \dot{\beta})
\end{aligned}
$$

From equation (5.1g) we are able solve for and make a global substitution for the quantity $Q^{2} e^{-6 \beta+\varphi+3 F+G}$. Making this substitution we have the following system of ODEs:

$$
\begin{aligned}
\ddot{\varphi}= & \frac{1}{4}\left(\dot{q}^{2}+\dot{G}^{2}-a^{2}+2(\dot{\varphi}+\dot{F})^{2}+12 \dot{\beta}^{2}-12 n^{2}+e^{2 \varphi+2 F+2 G}\left(l^{2}-\dot{\sigma}^{2}\right)\right) \\
\ddot{\beta}= & \frac{1}{4}\left(2 \dot{\varphi}^{2}-\dot{G}^{2}-\dot{q}^{2}-12 \dot{\beta}^{2}-2 \dot{F}^{2}-12 n^{2}-a^{2}-e^{2 \varphi+2 F+2 G}\left(l^{2}+\dot{\sigma}^{2}\right)\right) \\
& +\dot{\beta}(\dot{\varphi}+\dot{F}) \\
\ddot{\sigma}= & -\dot{\sigma}(\dot{\varphi}+\dot{F}+2 \dot{G}) \\
\ddot{q}= & \dot{q}(\dot{\varphi}+\dot{F}) \\
\ddot{F}= & \frac{1}{4}\left(3 a^{2}+\dot{q}^{2}+\dot{G}^{2}+36 n^{2}+12 \dot{\beta}^{2}+e^{2 \varphi+2 F+2 G}\left(l^{2}+3 \dot{\sigma}^{2}\right)-2(\dot{\varphi}-\dot{F})^{2}+8 \dot{F}^{2}\right)(5.2) \\
\ddot{G}= & \dot{G}(\dot{\varphi}+\dot{F})-e^{2 \varphi+2 F+2 G}\left(l^{2}-\dot{\sigma}^{2}\right) \\
& -\frac{1}{2}\left(2 \dot{\varphi}^{2}-\dot{G}^{2}-\dot{q}^{2}-12 \dot{\beta}^{2}-2 \dot{F}^{2}-12 n^{2}-a^{2}-e^{2 \varphi+2 F+2 G}\left(l^{2}+\dot{\sigma}^{2}\right)\right) \\
0= & a \dot{q}+l e^{2 \varphi+2 F+2 G} \dot{\sigma}+6 n\left(\dot{\varphi}+\dot{F}^{2}+2 \dot{\beta}\right)
\end{aligned}
$$


From the constraint (5.1g) we see that if $\dot{\varphi}=0$, then all of the other state variables must be simultaneously zero, which can only occur at an equilibrium point of the system. Hence $\dot{\varphi}$ must be positive (or negative) throughout the physical phase space. Here we shall assume $\dot{\varphi}>0$ (the case $\dot{\varphi}<0$ can be obtained by a time reversal - see later).

We define new variables of the form

$$
\begin{array}{lccc}
\tilde{F}=\frac{\dot{F}}{\dot{\varphi}} & \tilde{G}=\frac{1}{\sqrt{2}} \frac{\dot{G}}{\dot{\varphi}} \quad \tilde{q}=\frac{1}{\sqrt{2}} \frac{\dot{q}}{\dot{\varphi}} & \tilde{\sigma}=\frac{1}{\sqrt{2}} e^{\varphi+F+G} \frac{\dot{\sigma}}{\dot{\varphi}} \\
\tilde{\beta}=\sqrt{6} \frac{\dot{\beta}}{\dot{\varphi}} & \tilde{\Psi}_{1}=\sqrt{\frac{a^{2}+12 n^{2}}{2}} \frac{1}{\dot{\varphi}} & \tilde{\Psi}_{2}=\frac{1}{\sqrt{2}} e^{\varphi+F+G} \frac{l}{\dot{\varphi}}
\end{array}
$$

and a new time variable

$$
\frac{d t}{d \tau}=\frac{1}{\dot{\varphi}}
$$

The variables are chosen so that the transformed dynamical system has a compactified phase space. This property comes from the fact that $Q^{2} e^{-6 \beta+\varphi+3 F+G} \geq 0$ which implies that equation (5.1g) yields

$$
1 \geq \tilde{F}^{2}+\tilde{G}^{2}+\tilde{q}^{2}+\tilde{\sigma}^{2}+\tilde{\beta}^{2}+\tilde{\Psi}_{1}^{2}+\tilde{\Psi}_{2}^{2}
$$

The dynamical system (5.2) becomes

$$
\begin{aligned}
\frac{d \tilde{F}}{d \tau} & =\tilde{F}(2 \tilde{F}-\tilde{r})+\frac{1}{2}\left(\tilde{q}^{2}+\tilde{G}^{2}+\tilde{\beta}^{2}+3 \tilde{\sigma}^{2}+3 \tilde{\Psi}_{1}^{2}+\tilde{\Psi}_{2}^{2}-(1-\tilde{F})^{2}\right) \\
\frac{d \tilde{G}}{d \tau} & =\tilde{G}(1+\tilde{F}-\tilde{r})-\frac{1}{\sqrt{2}}\left(1-\tilde{F}^{2}-\tilde{G}^{2}-\tilde{q}^{2}-3 \tilde{\sigma}^{2}-\tilde{\beta}^{2}-\tilde{\Psi}_{1}^{2}+\tilde{\Psi}_{2}^{2}\right) \\
\frac{d \tilde{q}}{d \tau} & =\tilde{q}(1+\tilde{F}-\tilde{r}), \\
\frac{d \tilde{\sigma}}{d \tau} & =-\tilde{\sigma}(\sqrt{2} \tilde{G}+\tilde{r}), \\
\frac{d \tilde{\beta}}{d \tau} & =\tilde{\beta}(1+\tilde{F}-\tilde{r})+\frac{\sqrt{6}}{2}\left(1-\tilde{F}^{2}-\tilde{G}^{2}-\tilde{q}^{2}-\tilde{\sigma}^{2}-\tilde{\beta}^{2}-\tilde{\Psi}_{1}^{2}-\tilde{\Psi}_{2}^{2}\right), \\
\frac{d \tilde{\Psi}_{1}}{d \tau} & =-\tilde{\Psi}_{1} \tilde{r} \\
\frac{d \tilde{\Psi}_{2}}{d \tau} & =\tilde{\Psi}_{2}(1+\tilde{F}+\sqrt{2} \tilde{G}-\tilde{r}),
\end{aligned}
$$

and

$$
\tilde{r}=\frac{1}{2}\left[(1+\tilde{F})^{2}+\tilde{G}^{2}+\tilde{q}^{2}+\tilde{\beta}^{2}+\tilde{\Psi}_{2}^{2}-\tilde{\Psi}_{1}^{2}-\tilde{\sigma}^{2}\right],
$$

where the constraint equation becomes

$$
0=\sqrt{\frac{2}{a^{2}+12 n^{2}}} \tilde{\Psi}_{1}\left[\sqrt{2} a \tilde{q}+6 n\left(1+\tilde{F}+\frac{2}{\sqrt{6}} \tilde{\beta}\right)\right]+2 \tilde{\Psi}_{2} \tilde{\sigma} .
$$


There exists a first integral in the physical phase space $\left(\tilde{q} \neq 0, \tilde{\Psi}_{1} \neq 0\right)$ for this system. The function

$$
M=\frac{\tilde{\sigma} \tilde{\Psi}_{2}}{\tilde{q} \tilde{\Psi}_{1}}
$$

is constant, i.e., $M^{\prime}=0$. This implies a first integral for the original system of ordinary differential equations $(5.2$

$$
\dot{q}=C \dot{\sigma} e^{2 \varphi+2 F+2 G}
$$

where $C$ is a constant.

\section{Invariant Sets, Monotonic Functions}

We first recall that the phase space for this dynamical system is the interior and boundary of the compact set given by

$$
1 \geq \tilde{F}^{2}+\tilde{G}^{2}+\tilde{q}^{2}+\tilde{\sigma}^{2}+\tilde{\beta}^{2}+\tilde{\Psi}_{1}^{2}+\tilde{\Psi}_{2}^{2}
$$

Various hyperplanes divide the phase space into a number of different regions, they are $\tilde{q}=0, \tilde{\sigma}=0, \tilde{\Psi}_{1}=0$ and $\tilde{\Psi}_{2}=0$ hyperplanes. We note that $\tilde{\Psi}_{1}=0$ divides the phase space into two distinct regions $\tilde{\Psi}_{1}<0$ and $\tilde{\Psi}_{1}>0$. The dynamics in the invariant set $\tilde{\Psi}_{1}<0$ is the time reversal of the dynamics in the invariant set $\tilde{\Psi}_{1}>0$ (see (5.3)).

Consider the function

$$
M_{1}=\frac{\tilde{\sigma}^{2} \tilde{\Psi}_{2}^{2}}{\tilde{q}^{4}}
$$

and its derivative

$$
\frac{d M_{1}}{d \tau}=-2 M_{1}(1+\tilde{F})
$$

We easily see that this function is monotonically decreasing in the invariant set $\tilde{q} \neq 0, \tilde{\sigma} \neq$ $0, \tilde{\Psi}_{2} \neq 0$. Therefore, we can conclude that there are no closed or periodic orbits in the seven dimensional phase space, except possibly on the lower dimensional boundaries of this seven dimensional invariant set.

We restrict ourselves now to the invariant set $\tilde{\sigma}=0$. Consider the function

$$
M_{2}=\frac{\tilde{\Psi}_{1}^{2}}{\tilde{q}^{2}}
$$

and its derivative

$$
\frac{d M_{2}}{d \tau}=-2 M_{2}(1+\tilde{F})
$$


We easily see that this function is monotonically decreasing in the invariant set $\tilde{q} \neq 0, \tilde{\Psi}_{1} \neq 0$. Therefore, we can conclude that there are no closed or periodic orbits in this six dimensional phase space, except possibly on the lower dimensional boundaries of this six dimensional invariant set.

In the six dimensional invariant set $\tilde{\Psi}_{2}=0$, the function

$$
M_{3}=\frac{\tilde{\Psi}_{1}^{2}}{\tilde{q}^{2}}
$$

has the derivative

$$
\frac{d M_{3}}{d \tau}=-2 M_{3}(1+\tilde{F})
$$

which is monotonically decreasing in the set $\tilde{\Psi}_{2}=0, \tilde{q} \neq 0, \tilde{\Psi}_{1} \neq 0$. Therefore we conclude that there are no closed or periodic orbits in this six-dimensional invariant set.

In the six dimensional invariant set $\tilde{q}=0$, the function

$$
M_{4}=\frac{\tilde{\sigma}^{2} \tilde{\Psi}_{2}^{2}}{\tilde{\Psi}_{1}^{4}}
$$

has the derivative

$$
\frac{d M_{4}}{d \tau}=2 M_{4}(1+\tilde{F})
$$

which is monotonically increasing in the set $\tilde{q}=0, \tilde{\sigma} \neq 0, \tilde{\Psi}_{2} \neq 0$. Therefore we can conclude that there are no closed or periodic orbits in this six dimensional set.

With the existence of these monotonic functions $M_{1}, M_{2}, M_{3}$, and $M_{4}$, we can conclude that there are no closed or periodic orbits in the physical six dimensional phase space, [except possibly on lower dimensional (less than 5) invariant sets].

The zero-curvature spatially homogeneous and isotropic spacetimes are contained in the set $\tilde{q}=\tilde{\Psi}_{1}=\tilde{\Psi}_{2}=\tilde{G}-\sqrt{2} \tilde{F}=0$ union $\tilde{q}=\tilde{\Psi}_{1}=\tilde{\sigma}=\tilde{G}-\sqrt{2} \tilde{F}=0$.

The matter fields in (2.1) satisfy various energy conditions. For example, the positivity of the kinetic energy of the pseudo-axion scalar field, $\sigma$, demands that $\dot{\sigma}^{2}-l^{2} \geq 0$ (i.e., $\left.\tilde{\sigma}^{2}-\tilde{\Psi}_{2}^{2} \geq 0\right)$. However, we note that $\tilde{\sigma}^{2}-\tilde{\Psi}_{2}^{2}=0$ is not an invariant set.

\section{Equilibrium Points and Exact Solutions}

There are two equilibrium points and one three-dimensional equilibrium set.

- The three-dimensional equilibrium set is given by

$$
\left\{\tilde{F}^{2}+\tilde{G}^{2}+\tilde{q}^{2}+\tilde{\beta}^{2}=1, \tilde{\sigma}=0, \tilde{\Psi}_{1}=0, \tilde{\Psi}_{2}=0\right\}
$$


Note that since both $\tilde{\Psi}_{1}=0$ and $\tilde{\Psi}_{2}=0$ we necessarily have that $a=n=l=0$. At this point the value of $\tilde{r}=1+\tilde{F}_{0}$. The exact solution is then

$$
\begin{aligned}
\varphi(t) & =h_{1}-\frac{1}{\tilde{r}} \ln \left(\tilde{r} t+h_{0}\right), \\
F(t) & =F_{0}\left(h_{1}-\frac{1}{\tilde{r}} \ln \left(\tilde{r} t+h_{0}\right)\right)+F_{1} \\
G(t) & =\sqrt{2} G_{0}\left(h_{1}-\frac{1}{\tilde{r}} \ln \left(\tilde{r} t+h_{0}\right)\right)+G_{1} \\
q(t) & =\sqrt{2} q_{0}\left(h_{1}-\frac{1}{\tilde{r}} \ln \left(\tilde{r} t+h_{0}\right)\right)+q_{1} \\
\sigma(t) & =\sigma_{1} \\
\beta(t) & =\frac{1}{\sqrt{6}} \beta_{0}\left(h_{1}-\frac{1}{\tilde{r}} \ln \left(\tilde{r} t+h_{0}\right)\right)+\beta_{1}
\end{aligned}
$$

where $F_{0}^{2}+G_{0}^{2}+q_{0}^{2}+\beta_{0}^{2}=1$ and where $F_{1}, G_{1}, q_{1}, \sigma_{1}, \beta_{1}, h_{1}$ and $h_{0}$ are integration constants.

Since $a=n=l=0$, this metric is spatially homogeneous (and flat). Since $\tilde{\sigma}=0$ and $Q=0$ (which follows from the other conditions), this equilibrium set represents spatially flat solutions where the form-fields (the axion field and the four-form field strength) are trivial and only the dilaton and moduli fields are dynamically important. These solutions are known as the 'dilaton-moduli-vacuum' solutions (and their analytical form is given in [25]).

Recall that the dynamics of these models is restricted by the constraint given by equation (5.6). At these equilibrium points we are able to locally solve for the value of $\tilde{\Psi}_{1}$ and substitute into the remaining equations. The eigenvalues in the six-dimensional constraint surface are

$$
0,0,0, \sqrt{2} \tilde{G},-\sqrt{2} \tilde{G}-1-\tilde{F},-1+\tilde{F}+\sqrt{2} \tilde{G}-\sqrt{6} \tilde{\beta}
$$

The three zero eigenvalues correspond to the fact that this equilibrium set is threedimensional. It is clear that a subset of this equilibrium set will act as saddles of varying degree of stability, while another subset will act as sinks. Consequently, a subset of these dilaton-moduli-vacuum solutions with trivial form fields are sinks in the physical phase space, even in the presence of (negative) spatial curvature, and are thus generic attracting solutions.

- A second equilibrium point is

$$
\left\{\tilde{F}=-\frac{2}{3}, \tilde{G}=0, \tilde{q}=0, \tilde{\beta}=-\frac{1}{\sqrt{6}}, \tilde{\sigma}= \pm \frac{\sqrt{2}}{6}, \tilde{\Psi}_{1}=\frac{\sqrt{2}}{3}, \tilde{\Psi}_{2}=0\right\}
$$


Note that since $\tilde{\Psi}_{2}=0$ we have necessarily that $l=0$. At this point the value of $\tilde{r}=0$. [Note, since the dynamical system (5.5) is invariant under the transformation $\left(\tilde{\Psi}_{1}, \tilde{\Psi}_{2}\right) \rightarrow$ $\left(-\tilde{\Psi}_{1},-\tilde{\Psi}_{2}\right)$ there exists a corresponding equilibrium point with a $\tilde{\Psi}_{1}=-\sqrt{2} / 3$.] The exact solution is then

$$
\begin{aligned}
\varphi(t) & =h_{0} t+h_{1}, \\
F(t) & =-\frac{2}{3}\left(h_{0} t+h_{1}\right)+F_{1} \\
G(t) & =G_{1} \\
q(t) & =q_{1} \\
\sigma(t) & =\mp e^{-1 / 3\left(h_{0} t+h_{1}\right)-\left(F_{1}+G_{1}\right)}+\sigma_{1} \\
\beta(t) & =-\frac{1}{6}\left(h_{0} t+h_{1}\right)+\beta_{1}
\end{aligned}
$$

where $h_{0}=\frac{3}{2} \sqrt{a^{2}+12 n^{2}}, Q^{2}=\frac{1}{2}\left(a^{2}+12 n^{2}\right) e^{6 \beta_{1}-3 F_{1}-G_{1}}$ and $F_{1}, G_{1}, q_{1}, \sigma_{1}, \beta_{1}, h_{1}$ are all constants. In this situation the variable $\tilde{q}$ can be eliminated. The eigenvalues restricted to the constraint surface are

$$
\frac{1}{3}, \frac{1}{3}, \frac{1}{6}(1 \pm \sqrt{15+8 \sqrt{2}} i), \frac{1}{6}(1 \pm \sqrt{15-8 \sqrt{2}} i)
$$

This point represents a past attractor or a source. This corresponds to a spatially nonvacuum inhomogeneous model with a diagonal Einstein Tensor, having negative curvature.

The line element corresponding to this solution (after a few coordinate redefinitions) is

$$
d s^{2}=C^{2} e^{-2 \sqrt{a^{2}+12 n^{2}} t+6 n z}\left(-d t^{2}+d z^{2}\right)+\left(e^{a z} d x^{2}+e^{-a z} d y^{2}\right)
$$

- The third equilibrium point is

$$
\left\{\tilde{F}=-\frac{5}{7}, \tilde{G}=\frac{\sqrt{2}}{7}, \tilde{q}=0, \tilde{\beta}=-\frac{\sqrt{6}}{7}, \tilde{\sigma}=0, \tilde{\Psi}_{1}=\frac{2 \sqrt{3}}{7}, \tilde{\Psi}_{2}=0\right\}
$$

Note that since $\tilde{\Psi}_{2}=0$ we have necessarily that $l=0$. At this point the value of $\tilde{r}=0$. [Note, since the dynamical system (5.5) is invariant under the transformation $\left(\tilde{\Psi}_{1}, \tilde{\Psi}_{2}\right) \rightarrow$ $\left(-\tilde{\Psi}_{1},-\tilde{\Psi}_{2}\right)$ there exists a corresponding equilibrium point with a $\tilde{\Psi}_{1}=-2 \sqrt{3} / 7$.] The exact 
solution is then

$$
\begin{aligned}
\varphi(t) & =h_{0} t+h_{1}, \\
F(t) & =-\frac{5}{7}\left(h_{0} t+h_{1}\right)+F_{1} \\
G(t) & =\frac{2}{7}\left(h_{0} t+h_{1}\right)+G_{1} \\
q(t) & =q_{1} \\
\sigma(t) & =\sigma_{1} \\
\beta(t) & =-\frac{1}{7}\left(h_{0} t+h_{1}\right)+\beta_{1}
\end{aligned}
$$

where $h_{0}=\frac{7}{2 \sqrt{3}} \sqrt{a^{2}+12 n^{2}}, Q^{2}=\frac{1}{3}\left(a^{2}+12 n^{2}\right) e^{6 \beta_{1}-3 F_{1}-G_{1}}$ and $F_{1}, G_{1}, q_{1}, \sigma_{1}, \beta_{1}, h_{1}$ are all constants. This solution is a curved inhomogeneous model with a trivial axion field. In this situation the variable $\tilde{q}$ can be eliminated. The eigenvalues restricted to the constraint surface are

$$
\frac{2}{7}, \frac{2}{7}, \frac{4}{7},-\frac{2}{7}, \frac{1}{7}(1 \pm \sqrt{23} i)
$$

This point is always a saddle.

\section{DISCUSSION}

We have studied several classes of inhomogeneous string models whose governing equations reduce to ODE. In particular, we have found that generically solutions of the class of separable $G_{2}$ inhomogeneous M-theory cosmologies studied evolve from a spatially inhomogeneous and negatively curved model with a non-trivial form fields towards (a subset) of spatially flat and spatially homogeneous dilaton-moduli-vacuum solutions where the formfields (the axion field and the four-form field strength) are trivial and only the dilaton and moduli fields are dynamically important. This late time behaviour is the same as that of the spatially homogeneous models studied previously. However, in these models the inhomogeneities are not dynamically insignificant at early times, and the models asymptote (in the past) toward a new class of inhomogeneous cosmological models.

As noted earlier, the time-reversed dynamics of the $\dot{\varphi}>0$ models we have considered thus far is equivalent to the dynamics of the case where $\dot{\varphi}<0$. This follows by redefining the time variable according to $\frac{d t}{d \tau}=-\frac{1}{\dot{\varphi}}$ and appropriate definitions of the other state variables. The evolution equations will have an 'overall' change in sign, and hence the equilibrium 
points are identical in both cases, but the eigenvalues have opposite signs. Consequently, the dynamics of the $\dot{\varphi}<0$ models is the time reversal of the $\dot{\varphi}>0$ models and the timereversed dynamics of the above class of models is deduced by interchanging the sources and sinks and reinterpreting expanding solutions in terms of contracting ones, and vice-versa.

Although at late times (in the $\dot{\varphi}>0$ models) the inhomogeneities decay, the inhomogeneities are important at intermediate times and, in particular, at early times. Thus the qualitative features of the models are quite different to those of spatially homogeneous models studied previously. For example, in a study of FRW models [21] it was found that all negatively-curved FRW models evolve from the solution corresponding to a global source in which the curvature is (negative and) dynamically important (but with a trivial axion field) towards the dilaton-moduli-vacuum solutions [25], even in the presence of spatial curvature. The physical interpretation of these models, where both the NS-NS two-form potential and RR three-form potential are dynamically significant, was discussed in [21, with particular emphasis on the fact that the RR field causes the universe to collapse, but the NS-NS field has the opposite effect, whereby the interplay between these two fields leads to the models undergoing bounces. In the models under investigation here, orbits in the full phase space (with $\dot{\varphi}$ monotone) approach the dilaton-moduli-vacuum solution on the zero-curvature boundary (at late times) and again exhibit a 'bouncing' behaviour; this bouncing behaviour is the result of the orbits shadowing orbits in the boundary that are constantly being redirected to saddle points of the same or higher stability until it reaches a stable equilibrium.

\section{Acknowledgments}

Both AAC and RJvdH are supported by research grants through Natural Sciences and Engineering Research Council of Canada. RJvdH wishes to acknowledge the support of the University Council on Research at St. Francis Xavier University.

\section{References}

[1] M. B. Green, J. H. Schwarz, and E. Witten, Superstring Theory, in 2 vols., (Cambridge University Press, Cambridge, 1987); J. Polchinski, String Theory, in 2 vols., (Cambridge 
University Press, Cambridge, 1998).

[2] E. Witten, Nucl. Phys. B443, 85 (1995).

[3] K. Tomita, Prog. Theor. Phys. 59, 1150 (1978).

[4] G. Veneziano, Phys. Lett. B406, 297 (1997), hep-th/9703150.

[5] J. Maharana, E. Onofri, and G. Veneziano, J. High Energy Phys. 01, 004 (1998), grqc/9802001.

[6] V. A. Belinskii and I. M. Khalatnikov, Sov. Phys. JETP 29, 911 (1969).

[7] V. A. Belinskii and I. M. Khalatnikov, Sov. Phys. JETP 30, 1174 (1970).

[8] V. A. Belinskii and I. M. Khalatnikov, Sov. Phys. JETP 32, 169 (1971).

[9] V. A. Belinskii, E. M. Lifshitz, and I. M. Khalatnikov, Adv. Phys. 31, 639 (1982).

[10] J. D. Barrow and K. Kunze, Phys. Rev. D56, 741 (1997), hep-th/9701085.

[11] A. Feinstein, R. Lazkoz, and M. A. Vazquez-Mozo, Phys. Rev. D56, 5166 (1997), hepth/9704173.

[12] D. Clancy, A. Feinstein, J. E. Lidsey, and R. Tavakol, Phys. Rev. D60, 043503 (1999), grqc/9901062.

[13] I. Bakas, Nucl. Phys. B428, 374 (1994), hep-th/9402016.

[14] J. Maharana, Phys. Rev. Lett. 75, 205 (1995), hep-th/9502002.

[15] A. A. Kehagias, Phys. Lett. B360, 19 (1995), hep-th/9506205.

[16] R. Geroch, J. Math. Phys. 13, 394 (1972).

[17] J.E. Lidsey, D. Wands and E. Copeland, Phys. Rept. 337 (2000), hep-th/9909061.

[18] J. Wainwright, W. C. W. Ince, and B. J. Marshman, Gen. Rel. Grav. 10, 259 (1979).

[19] M. Carmeli, Ch. Charach, and A. Feinstein, Ann. Phys. 150, 392 (1983).

[20] A. P. Billyard, A. A. Coley, and J. E. Lidsey, Phys. Rev. D. 59, 123505 (1999); ibid, J. Math. Phys. 40, 5092 -5105 (1999); ibid, Class. Quant. Grav. 17, 453-484 (2000).

[21] A. P. Billyard, A. A. Coley, J. E. Lidsey and U. S. Nilsson, Phys. Rev D 61, 043504 (2000).

[22] A. Feinstein and J. Ibanez, Class. Quantum Grav., 10, L227-L231, (1993)

[23] N. Kaloper, I. I. Kogan, and K. A. Olive, Phys. Rev. D57, 7340 (1998); Erratum, ibid. D60, 049901 (1999).

[24] A. P. Billyard, Ph. D thesis, Dalhousie University (1999)

[25] E. J. Copeland, A. Lahiri, and D. Wands, Phys. Rev. D50, 4868 (1994). 